$\Rightarrow$ MUCOSALIMMUNOLOGY

\section{Probiotic induction of tolerogenic T cells in the gut}

Upon reaching the small intestinal epithelium, lamina propria $\mathrm{CD} 4^{+}$ $\mathrm{T}$ cells can reactivate the CD8 $\mathrm{T}$ cell lineage programme, in part by downregulating levels of the transcription factor THPOK, to differentiate into $\mathrm{CD} 4{ }^{+} \mathrm{CD} 8 \alpha \alpha^{+} \alpha \beta$ T cells (also known as double-positive intraepithelial lymphocytes (DP IELs)), which promote tolerance to dietary antigens. Cervantes-Barragan et al. now show that a species of probiotic bacteria,
Lactobacillus reuteri, induces the differentiation of $\mathrm{CD} 4^{+} \mathrm{T}$ cells into DP IELs by activating their aryl hydrocarbon receptor (AHR), leading to a decrease in THPOK levels.

The authors observed that DP IELs were present in mice from Charles River Laboratories (CR), but not in those from the Jackson laboratories (JAX), and that the DP IEL-positive phenotype could be transferred to JAX mice by colonizing them with ileal or faecal microbiota from CR mice. Treating CR mice with antibiotics that target Gram-positive bacteria abrogated DP IEL development, suggesting that DP IELs are induced by Gram-positive bacteria in the gut. Indeed, $L$. reuteri was present in the ilea of CR but not JAX mice, and two strains of $L$. reuteri induced DP IELs in JAX mice.

L. reuteri metabolizes dietary tryptophan to indole derivatives that activate AHR, and the authors found that cell-free supernatant from L. reuteri grown in tryptophancontaining medium stimulated the differentiation of $\mathrm{CD} 4^{+} \mathrm{T}$ cells into DP IELs, as did an AHR agonist. By contrast, an AHR antagonist inhibited the differentiation of $\mathrm{CD} 4^{+} \mathrm{T}$ cells into DP IELs. These data suggest that the L. reuteri supernatant acts through AHR. Indeed, a L. reuteri mutant that cannot metabolize tryptophan into AHR ligands could not induce DP IELs in JAX mice, and the frequency of DP IELs was reduced in mice harbouring $\mathrm{Ahr}^{--} \mathrm{T}$ cells compared with that in wild-type littermates. Further data suggested that the absence of AHR blocks the differentiation of IELs into DP IELs upstream of THPOK downregulation.

In summary, $L$. reuter $i$ induces DP IELs by activating AHR, leading to downregulation of THPOK. Of note, although a high-tryptophan diet increased the frequency of DP IELs in CR mice, it could not induce DP IELs in germ-free mice monocolonized with L. reuteri, suggesting that $L$. reuteri acts as part of the microbiota to induce DP IELs in the presence of tryptophan.

Katharine H. Wrighton

ORIGINAL ARTICLE Cervantes-Barragan, L. et al. Lactobacillus reuteri induces gut intraepithelial $\mathrm{CD}^{+}{ }^{+} \mathrm{CD} 8 \mathrm{a \alpha}^{+} \mathrm{T}$ cells. Science 357, 806-810 (2017) 